\title{
Neumonía a repetición en un paciente con deformidades óseas: a propósito de un caso de raquitismo
}

\author{
Recurrent pneumonia in a patient with bone deformity: a case report of ricketts \\ Carlos Andrés Zapata-Chica ${ }^{1, a}$, Juan José Ramírez ${ }^{2, b, c}$, María Fernanda Rodríguez-Ballesteros ${ }^{2, d}$, Daniela \\ Franco-Herrera ${ }^{2, d}$
}

\section{RESUMEN}

El raquitismo es una enfermedad ósea infrecuente asociada a la disminución de niveles séricos de calcio o fosfato, la mayoría de los casos como consecuencia de déficit nutricional, aunque puede presentarse por mutaciones genéticas o defectos adquiridos del metabolismo. El cuadro clínico es heterogéneo y depende de la edad de presentación; manifestándose con ensanchamiento y retraso de la mineralización de la placa de crecimiento ósea. Se presenta el caso de un niño indígena de 11 meses de edad con historia de neumonía recurrente, al examen físico, presentaba hipotonía muscular generalizada, tórax con rosario costal y deformidad en extremidades, con paraclínicos: hipocalcemia e hipofosfatemia, fosfatasa alcalina y hormona paratiroidea elevadas, niveles de 25-hidroxi-vitamina $\mathrm{D}_{3}$ normales-altos y con valor de 1,25-dihidroxi-vitamina $\mathrm{D}_{3}$ muy bajo, se sugirió el diagnóstico de raquitismo dependiente de vitamina D tipo 1A severo, una forma infrecuente de esta patología.

PALABRAS CLAVE: Raquitismo, preescolar, vitamina D. (Fuente: DeCS BIREME).

\section{SUMMARY}

Ricketts is a rare bone disease associated with low serum levels of calcium and phosphate. Most of the cases occur as a consequence of nutritional deficits, but genetic mutations or acquired metabolic defects may account for it. Clinical presentation is heterogenous depending on age at presentation, manifesting as widening and retardation of mineralization of the growing bone plate. We present the case of a 11 month of age child with a history of recurrent pneumonia presenting with generalized muscle hypotonia, deformed chest and bowed extremities with hypocalcemia and hypophosphatemia, elevated levels of alkaline phosphatase and parathyroid hormone, high levels of 25-hidroxy-vitamine D3 and very low levels of 1,25-dihidroxy-vitamine D3 suggesting the diagnosis of severe vitamin D type 1 A rickets, a rare form of this disease.

KEYWORDS: Rickets, child preschool, vitamin D. (Source: MeSH NLM).

1. Hospital Universitario San Jorge. Pereira, Risaralda, Colombia.

2. Universidad Tecnológica de Pereira. Pereira, Risaralda, Colombia.

a. Nefrólogo Pediatra;

b. Endocrinólogo Pediatra;

c. Docente;

d. Médico general 


\section{INTRODUCCIÓN}

El raquitismo es una enfermedad ósea caracterizada por alteración en la mineralización normal del hueso o del tejido osteoide en crecimiento. El cuadro clínico es heterogéneo y depende de la edad de presentación y patogénesis, pero incluye arqueamiento de las extremidades (genu varu o valgo), talla baja, debilidad muscular, dolores óseos, ensanchamiento metafisiario, fracturas patológicas y retardo en la dentición $(1,2)$. El trastorno es generado principalmente por deficiencias nutricionales. Los defectos genéticos, los defectos adquiridos en el metabolismo de la vitamina D o los defectos en la reabsorción tubular de minerales, son causas menos frecuentes (3). Las mutaciones en las proteínas involucradas en el metabolismo de estos nutrientes se han descrito como etiologías raras del raquitismo ( $13 \%$ de los casos), y se dividen en dos grupos: raquitismo calciopénico, denominados dependientes de vitamina $\mathrm{D}, \mathrm{y}$ raquitismo hipofosfatémico (4-5).

El raquitismo nutricional se presenta principalmente en países en desarrollo como Colombia, donde, sin embargo, la implementación de estrategias para su control ha disminuido la prevalencia. En este artículo presentamos un caso infrecuente de raquitismo dependiente de vitamina $\mathrm{D}$, probablemente tipo $1 \mathrm{~A}$, en un niño de 11 meses de edad que fue remitido a nuestra institución por neumonía severa a repetición. Describimos cómo las deformidades óseas pueden sugerir la presencia de otra patología subyacente como factor de riesgo para las infecciones respiratorias recurrentes, y resaltamos el inicio oportuno de estudios de imagen y metabólicos, para diagnosticar otras variedades de raquitismo menos frecuentes.

\section{PRESENTACIÓN DEL CASO}

Niño de 11 meses de edad, indígena Embera Chamí, con antecedente de neumonía a repetición. Fue producto de primer embarazo de madre adolescente de 14 años, con parto a término y peso adecuado. Padres consanguíneos en tercer grado. Ingresó por cuadro clínico de dificultad respiratoria severa que requirió manejo con ventilación mecánica y antibiótico en la unidad de cuidado crítico. Al examen físico se evidenció desnutrición proteico-calórica, su peso fue $6,8 \mathrm{~kg}$ (valor $\mathrm{z}<-3$ ) y talla: $68 \mathrm{~cm}$ (valor $\mathrm{z}<$ -3). Presentaba hipotonía muscular generalizada, incluyendo músculos de la deglución, tórax con rosario costal bilateral, deformidad en el fémur derecho y tercio distal de ambos antebrazos, y ensanchamiento de las muñecas (figura 1). Con respecto a la dentición tenía los dos incisivos centrales inferiores y estaban en erupción los dos incisivos centrales superiores.

Recibía lactancia materna y desde los 8 meses, alimentación complementaria. No habían referidos síntomas gastrointestinales. Las radiografías óseas mostraron fracturas de radio y cúbito bilateral (figuras $2 \mathrm{~B}$ y $2 \mathrm{C}$ ), y fracturas en ambos fémures (figura 2A). Se evidenció, además, alteración en la densidad ósea generalizada y ensanchamiento de las metáfisis en forma de copa, con deshilachamiento distal (figura 2).
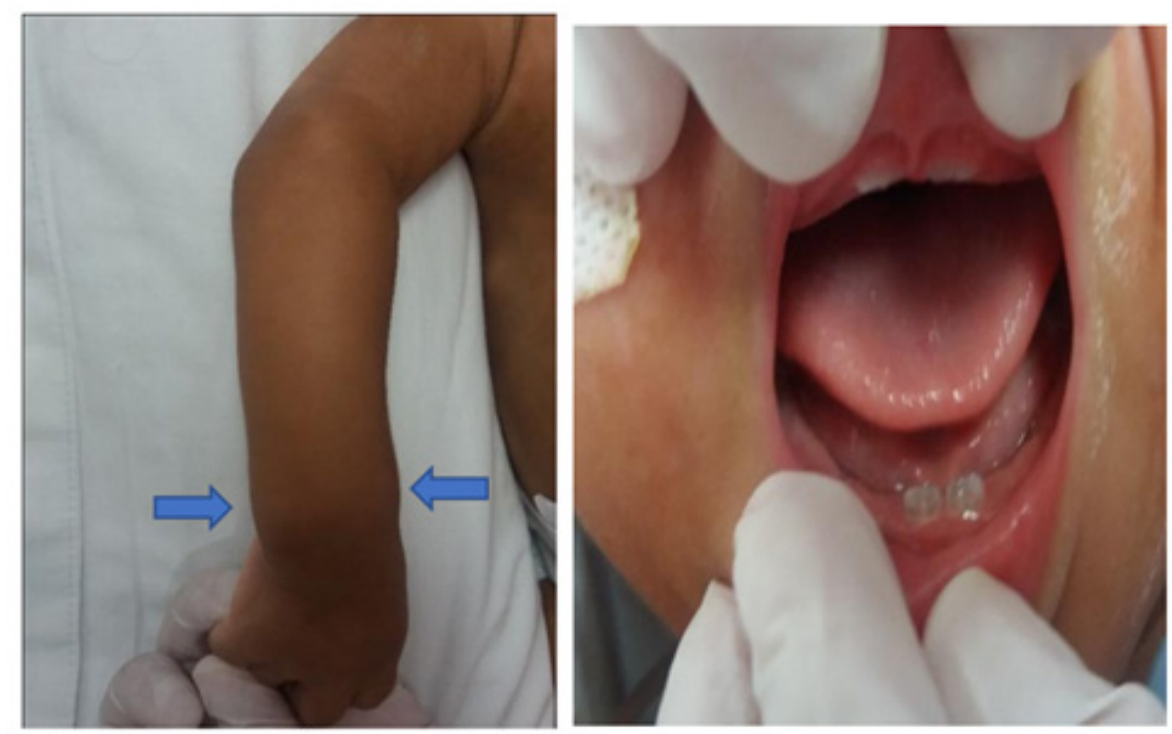

Figura 1. Ensanchamiento de las muñecas (flechas azules), Incisivos inferiores con dentina pobremente mineralizada. 

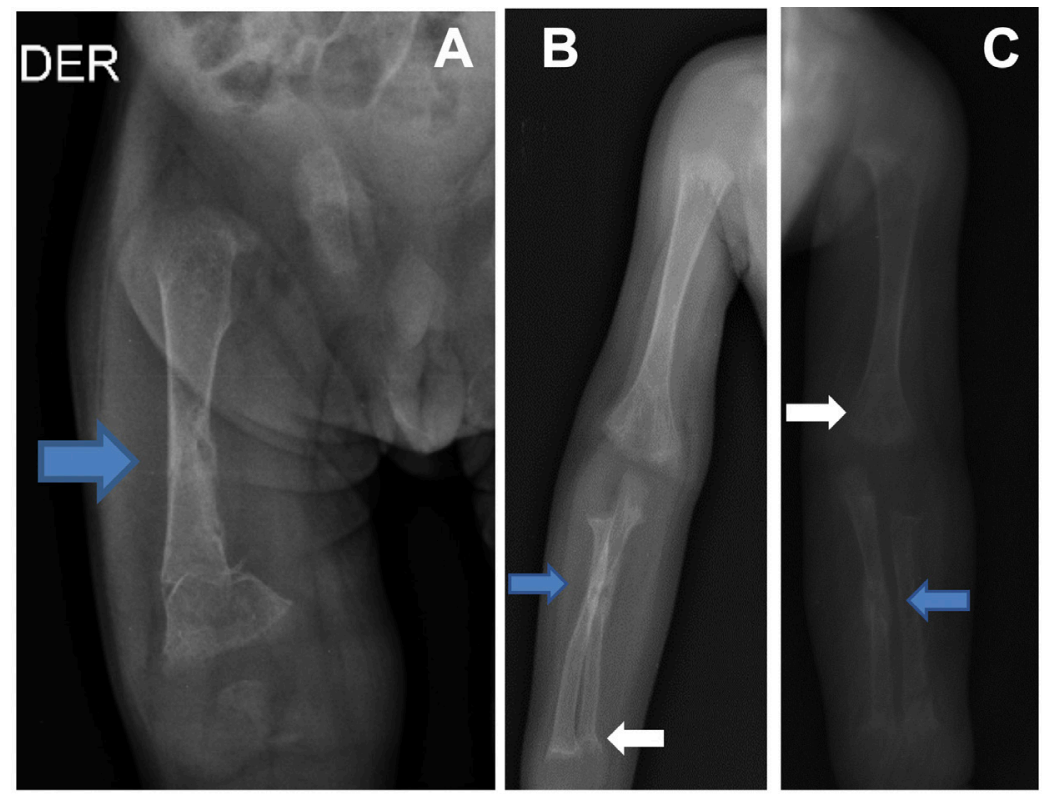

Figura 2. Radiografías óseas. Osteopenia difusa, irregularidades de las metáfisis con deshilachamiento y evidencia de "copa invertida" (flechas blancas). A. Fractura de fémur derecho. B y C. Fracturas de cúbito y radio bilateral (flechas azules).

Tabla 1. Exámenes de laboratorio bioquímico.

\begin{tabular}{lcc}
\hline Laboratorio & Resultado & Valor normal \\
\hline Sodio & 136 & $135-145 \mathrm{mmol} / 1$ \\
Potasio & 3,5 & $3,5-5 \mathrm{mmol} / 1$ \\
Cloro & 98 & $98-105 \mathrm{mmol} / 1$ \\
Magnesio & 1,6 & $1,6-2,4 \mathrm{mg} / \mathrm{dl}$ \\
Calcio iónico & 0,67 & $0,9-1,2 \mathrm{mmol} / 1$ \\
Fósforo & 2,4 & $3,5-5 \mathrm{mg} / \mathrm{dl}$ \\
Fosfatasa alcalina & 1108 & $156-369 \mathrm{U} / 1$ \\
PTH & 424 & $10-55 \mathrm{pg} / \mathrm{ml}$ \\
$1,25-(\mathrm{OH})_{2 \mathrm{D}}{ }^{3}$ & 8 & $15-60 \mathrm{pg} / \mathrm{ml}$ \\
$25(\mathrm{OH})_{\mathrm{D}}{ }^{3}$ & 52 & $17-54 \mathrm{ng} / \mathrm{ml}$ \\
HCO $_{3}$ & 22,7 & $>18 \mathrm{mEq} / 1$ \\
Albúmina & 3,4 & $3,5-5 \mathrm{~g} / \mathrm{dl}$ \\
Creatinina & 0,2 & $0,2-0,5 \mathrm{mg} / \mathrm{dl}$ \\
\hline $1,25-(\mathrm{OH})_{2} \mathrm{D}_{2}: 1,25$ dihidroxi Vitamina $\mathrm{D}_{3}$ & \\
$25(\mathrm{OH})_{\mathrm{D} 3:} 25$ hidroxi Vitamina $\mathrm{D}_{3}$ &
\end{tabular}

En los exámenes de laboratorio bioquímicos, se encontró hipocalcemia e hipofosfatemia, fosfatasa alcalina y hormona paratiroidea elevadas, y niveles de 25 hidroxi vitamina $\mathrm{D}_{3}$ normal-alto, y de 1,25 dihidroxi vitamina $\mathrm{D}_{3}$ muy bajo. La creatinina, hemoglobina, glucosa, bilirrubinas, transaminasas y el perfil tiroideo fueron normales (tabla 1). El uroanálisis no mostró alteraciones; la excreción urinaria de calcio era normal (índice calcio/creatinina 0,35; valor normal para su edad $(<0,6)$; y la reabsorción tubular de fósforo baja (53\%) valor normal $>85 \%)$. La ecografía renal fue normal, sin nefrocalcinosis.

Se inició tratamiento con suplementación de calcio y fósforo, inicialmente por vía parenteral dadas sus condiciones críticas, con posterior administración adicional de calcitriol. Luego de la evaluación por el servicio de ortopedia, se procedió con inmovilización de miembros inferiores. La estancia hospitalaria fue prolongada, pero salió sin complicaciones, con niveles séricos de calcio y fósforo normales (calcio iónico: $0,92 \mathrm{mmol} / \mathrm{l}$ y fósforo: $3,9 \mathrm{mg} / \mathrm{dl}$ ).

$\mathrm{Al}$ alta, continuó suplementación oral de calcio: 50 $\mathrm{mg} / \mathrm{kg}$ /día, fósforo $35 \mathrm{mg} / \mathrm{kg} /$ día y calcitriol: $0,03 \mathrm{mcg} /$ $\mathrm{kg} / \mathrm{d}$ á. Se dio orden de seguimiento ambulatorio por nefrología y endocrinología pediátricas, y ortopedia; no obstante, el paciente no registra asistencia a los mismos en nuestra institución.

\section{DISCUSIÓN}

El raquitismo es una enfermedad ósea que está asociada a niveles séricos bajos de calcio o fósforo, lo que lleva a una disminución y retardo en la mineralización de las placas de crecimiento en los 
huesos(6), como se evidenció en el examen físico de nuestro paciente.

$\mathrm{Al}$ ser un niño indígena y presentar además signos clínicos de desnutrición, se consideró como primera causa un raquitismo carencial, dado que este tipo sigue siendo el más frecuente (7). Sin embargo, con la disminución en su prevalencia global (8), las formas genéticas han llegado a ser proporcionalmente más comunes, por lo que no se descartó esta posibilidad etiológica.

La severidad del cuadro, el antecedente de consanguinidad en sus padres y sus niveles normales de $25(\mathrm{OH}) \mathrm{D}_{3}$, nos sugerían otras variantes incluidas las genéticas. Un síndrome de malabsorción era improbable por los niveles normales de $25(\mathrm{OH})$ $\mathrm{D}_{3}$ y la ausencia de síntomas gastrointestinales; no se solicitaron anticuerpos anti endomisio ni anti transglutaminasa. Se descartó síndrome de Fanconi por la ausencia de otras pérdidas tubulares (9). Entre otros, no se encontró glucosuria, hipercalciuria ni acidosis metabólica.

Considerando una etiología genética, la hipocalcemia severa y el hiperparatiroidismo en el paciente descartaban la posibilidad de la hipofosfatemia familiar ligada al cromosoma X (10-11). Finalmente, la hipocalcemia e hipofosfatemia simultáneas, con valores normales-elevados de $25(\mathrm{OH}) \mathrm{D}_{3}$ y muy bajos de $1,25(\mathrm{OH})_{2} \mathrm{D}_{3}$ nos sugirieron un raquitismo dependiente de vitamina $\mathrm{D}$ tipo $1 \mathrm{~A}$, aunque no se pudo hacer la confirmación genética, ya que no disponíamos de la prueba.

La debilidad muscular, incluidos los músculos de la deglución, secundaria al déficit de calcio y fósforo, y la escasa mineralización de la caja torácica, suponen una susceptibilidad para adquirir infecciones respiratorias frecuentes como sucedió en nuestro paciente $(1,3)$.

El tratamiento recomendado para este tipo raquitismo es la administración oral de dosis de reemplazo fisiológicas de $1,25(\mathrm{OH})_{2} \mathrm{D}_{3}$ (calcitriol), en un rango de 10 a $400 \mathrm{ng} / \mathrm{kg} /$ día de por vida (3). Descrito por otros autores, algunos pacientes también requieren inicialmente la suplementación de calcio y fósforo mientras se estabilizan sus niveles séricos $(1,5,12)$, acorde con la conducta terapéutica realizada en nuestro paciente.

En conclusión, debido a la singularidad de la patología presentada por el paciente, pudimos observar que las manifestaciones clínicas e imagenológicas en los diferentes tipos de raquitismo pueden ser muy similares, incluidas las deformidades óseas. Son los exámenes de laboratorio los que permiten hacer un diagnóstico diferencial. Sospechar oportunamente las variantes menos frecuentes de raquitismo y conocer sus diferentes perfiles bioquímicos, permitirán un diagnóstico y tratamiento adecuado de los niños afectados.

\section{Declaración de financiamiento y de conflictos de interés:}

Los autores declaran no tener conflictos de interés. El reporte fue financiado por los autores.

\section{Contribución de autoría:}

CAZC: Concepción y diseño del trabajo, Análisis e interpretación de datos, revisión crítica del manuscrito. JJRJ: Análisis e interpretación de datos, Revisión crítica del manuscrito. DFH: Recolección y obtención de datos, Redacción del manuscrito. MFRB: Recolección y obtención de datos, Redacción del manuscrito.

\section{Correspondencia:}

Maria Fernanda Rodriguez Ballesteros

Carrera 35bis \#25-314 manzana 7 casa 34, Dosquebradas, Risaralda, Colombia.

Correo electrónico: mariarodriguez@utp.edu.co

\section{REFERENCIAS BIBLIOGRÁFICAS}

1. Rocha A. Diagnóstico diferencial del raquitismo hipocalcémico: caso clínico. Rev chil pediatr. 2013; 84(6): 672-80. DOI: 10.4067/S037041062013000600011

2. Elder CJ, Bishop NJ. Rickets. Lancet. 2014; 383(9929):1665-1676. DOI: $10.1016 / \mathrm{S} 0140-$ 6736(13)61650-5

3. Carpenter TO, Shaw NJ, Portale AA, Ward LM, Abrams SA, Pettifor JM. Rickets. Nat Rev Dis Primers. 2017; 3:17101. DOI: 10.1038/nrdp.2017.101

4. Ramon-Krauel M. Raquitismo de causa genética. Rev Esp Endocrinol Pediatr. 2018; 9(1):48-53. DOI: 10.3266/RevEspEndocrinolPediatr.pre201

5. Halty $M$, Caggiani $M$. Raquitismo vitamina $D$ dependiente tipo I.Arch Pediatr Urug. 2006;77(2):1607.

6. Acar S, Demir K, Shi Y. Genetic causes of rickets. J Clin Res Pediatr Endocrinol. 2017; 9(Suppl 2):88105. DOI: $10.4274 /$ jcrpe.2017.S008 
7. Munns CF, Shaw N, Kiely M, Specker BL, Thacher TD, Ozono $\mathrm{K}$, et al. Global consensus recommendations on prevention and management of nutritional rickets. Horm Res Paediatr. 2016;85(2):83106. ISSN: 1663-2818.

8. Creo AL, Thacher TD, Pettifor JM, Strand MA, Fischer PR. Nutritional rickets around the world: an update. Paediatr Int Child Health. 2017;37(2):8498. DOI: 10.1080/20469047.2016.1248170

9. Zúñiga M, Galindo A, Galaz MI, Vivanco M, Romero $\mathrm{P}$, Balboa $\mathrm{P}$, et al. Síndrome de Fanconi y raquitismo hipofosfatémico asociado al uso de tenofovir en una niña infectada con VIH. Rev chil pediatr. 2017;88(1):148-52. DOI: 10.1016/j.rchipe.2016.08. 001
10. Hernández GG, Belmont Laguna F. Dental characteristics of hypophosphatemic rickets. Case report. Rev Odonto Mex. 2013;17(2):103-10.

11. Pavone V, Testa G, Gioitta Iachino S, Evola FR, Avondo S, Sessa G. Hypophosphatemic rickets: etiology, clinical features and treatment. Eur J Orthop Surg Traumatol. 2015;25(2):221-6. DOI: 10.1007/ s00590-014-1496-y.

12. Velásquez-Jones L, Medeiros M, Valverde-Rosas S, Jiménez-Triana C, del Moral-Espinosa I, RomoVázquez JC, et al. Seguimiento a largo plazo de un paciente con raquitismo dependiente de vitamina D tipo I. Bol Med Hosp Infant Mex. 2015;72(3):190-4.

Recibido: 01/07/2019

Aceptado: 19/09/2019 